\title{
Limitação da emissão de gases de efeito estufa, desmatamento e crescimento econômico no Brasil: uma análise prospectiva
}

Submissão: $15 / 03 / 2021$

Aceite: $24 / 06 / 2021$

Benedito Silva Neto'

\begin{abstract}
Resumo
De acordo com o Painel Internacional sobre Mudança Climática (IPCC em inglês) o aquecimento global, pelas suas repercussões sobre o clima, representa uma séria ameaça à Humanidade. $O$ objetivo do artigo é analisar as restrições ao crescimento econômico do Brasil colocadas pelas metas propostas pelo IPCC em 2014 para a limitação do aquecimento global, considerando a importância das emissões provocadas pelo desmatamento. Após a introdução, na segunda seção é realizada uma análise da emissão de gases de efeito estufa (GEE) no Brasil entre 1990 e 2018. $\mathrm{Na}$ terceira seção é apresentado um modelo que relaciona a emissão de GEE com o Produto Interno Bruto (PIB) e a com a população. Na quarta seção são apresentados os cenários de crescimento econômico. Na quinta seção são discutidos os resultados das simulações realizadas por meio dos cenários. Conforme esses resultados, para atingir a meta do IPCC estabelecida em 2014, considerando a eliminação do desmatamento até 2050 a uma taxa constante, o conteúdo de carbono (toneladas de $\mathrm{CO}_{2}$ equivalente por PIB) da economia brasileira (descontado o desmatamento) deveria ser reduzido a $57,44 \% ; 41,27 \% ; 30,48 \%$ e $22,31 \%$ em relação a 2018 , considerando, respectivamente, um crescimento do PIB por habitante de $0 \%, 1 \%, 2 \%$ e $3 \%$ ao ano, e um crescimento populacional anual de $0,35 \%$. Conclui-se que a total eliminação do desmatamento no Brasil até 2050 é uma condição praticamente incontornável para que o país possa ter um crescimento econômico compatível com as metas de redução das emissões de GEE estabelecidas pelo IPCC em 2014.
\end{abstract}

Palavras-chave: Aquecimento Global. Mudança Climática. Identidade de Kaya. Desmatamento.

\section{Limitation of greenhouse gas emissions, deforestation and economic growth in Brazil: a prospective analysis}

\begin{abstract}
According to the International Panel on Climate Change (IPCC), global warming, due to its repercussions on the climate, represents a serious threat to humanity. The objective of the article is to analyze the restrictions to economic growth in Brazil posed by the goals proposed by the IPCC in 2014 to limit global warming, considering the importance of emissions caused by deforestation. After the introduction, in the second section an analysis of the emission of greenhouse gases (GHG) in Brazil between 1990 and 2018 is carried out. In the third section a model is presented that relates the GHG emission with the Gross Domestic Product (GDP) and with the population. In the fourth section, economic growth scenarios are presented. In the fifth section, the results of the simulations performed through the scenarios are discussed. According to these results, in order to reach the IPCC target established in 2014, considering the elimination of deforestation by 2050 at a constant rate, the carbon content (tons of CO2 equivalent per GDP) of the Brazilian economy (discounting deforestation) should be reduced to $57.44 \% ; 41.27 \%$; $30.48 \%$ and $22.31 \%$ in relation to 2018, considering, respectively, a GDP growth per inhabitant of $0 \%, 1 \%, 2 \%$ and $3 \%$ per year, and an annual population growth of $0.35 \%$. It is concluded that the total elimination of deforestation in Brazil by 2050 is a practically unavoidable condition for the country to have an economic growth compatible with the goals of reducing GHG emissions established by the IPCC in 2014.
\end{abstract}

Keywords: Global Warming. Climate Change. Kaya Identity. Deforestation.

\footnotetext{
${ }^{1}$ Doutorado em Agricultura Comparada e Desenvolvimento Agrícola (INA-Paris-Grignon/França). Pós-doutorado no Institut des Sciences et Industries du Vivant et de l'Environment (Paris/França). Professor do Programa de PósGraduação em Desenvolvimento e Políticas Públicas da Universidade Federal da Fronteira Sul (UFFS). https://orcid.org/0000-0001-8497-0124 E-mail: bsilva@uffs.edu.br
} 


\section{Introdução}

Em 1990 o Painel Internacional sobre Mudança Climática (sigla em inglês IPCC) apresentou um relatório com estratégias para responder à mudança climática (IPCC, 1990). Fruto do trabalho de centenas de estudiosos de diferentes áreas do conhecimento, esse relatório já mostrava claramente os enormes riscos que representa a emissão de gases de efeito estufa gerada pelas atividades humanas, na medida em que esta emissão pode provocar um aquecimento global da atmosfera com efeitos sobre o clima que podem ter consequências catastróficas para a Humanidade. A partir de então as pesquisas sobre o aquecimento global e suas consequências se intensificaram, envolvendo milhares de pesquisadores de todo o mundo, resultando em uma grande quantidade de dados e interpretações. Um intenso debate público se estabeleceu. Ao longo deste debate, a mudança climática tornou-se um tema de primeiro plano na agenda política internacional.

Após trinta anos do início deste movimento, as evidências de um aquecimento global de origem antrópica, assim como dos seus efeitos sobre o clima do planeta, são dificilmente contestáveis. Um aumento da temperatura de 1으 C em todo o planeta, e de 1,5으 C no Brasil, entre 1850 e 2010 já é evidenciado cientificamente (ARTAXO, 2014). Tais aumentos têm provocado efeitos importantes sobre o clima, evidenciados por fenômenos como deslocamentos e decréscimos incomuns da população de várias espécies animais e vegetais, e intensificação de eventos climáticos extremos, como secas, furacões e tornados (ARTAXO, 2014). Diante desta situação, coloca-se a questão de qual seria o nível de atividade econômica compatível com uma limitação do aquecimento global que permita evitar a intensificação dos efeitos da mudança climática.

O objetivo deste artigo é analisar as possibilidades de crescimento econômico do Brasil diante das metas propostas pelo IPCC (2014) para a limitação do aquecimento global, considerando a grande importância do desmatamento para a emissão de GEE no país. Metodologicamente, esta análise se baseia em simulações realizadas a partir de uma versão da Identidade de Kaya (KAYA, 1990). Esta identidade tem sido largamente empregada para a análise das relações entre o setor de energia da economia e a emissão de gases de efeito estufa (FEIJÓ; RANGEL, 2018; JANSSENS-MANHOUT, G.; PAROULO, P.; MARTELLI, S., 2013). Neste artigo, a partir dos mesmos pressupostos empregados para a formulação da Identidade de Kaya é formulada uma identidade do mesmo tipo na qual são expressos todos os setores responsáveis pela emissão de GEE considerados pelo Sistema de Estimativas de Gases de Efeito Estufa (SEEG, 2019), com 
destaque para o setor de Mudanças no Uso da Terra, no qual o desmatamento é considerado. Esta versão é denominada "Identidade de Kaya Composta" (IKC).

O presente artigo está organizado em seis seções. A primeira seção corresponde a esta introdução. Na segunda seção é realizada uma análise das fontes de emissão de gases de efeito estufa (GEE) no Brasil entre 1990 e 2018. Na terceira seção é discutida a formulação da IKC, e na quarta são apresentados os cenários a serem analisados por meio dela. Na quinta seção são discutidos os resultados das simulações realizadas a partir dos cenários, considerando os dados apresentados nas seções anteriores. Na última seção são apresentadas as conclusões.

\section{As emissões de GEE no Brasil}

De maneira geral, o debate sobre a promoção de ações para a limitação das emissões de GEE se concentram mais na questão energética. Neste debate, a substituição dos combustíveis fósseis por fontes de energia renováveis em geral é considerada como a principal medida para diminuir a emissão de GEE. No entanto, há outras fontes de emissão de GEE que, em muitos casos, como no Brasil, geram quantidades maiores destes gases do que os combustíveis fósseis. Um aspecto interessante da classificação das fontes de emissão de GEE pelo Sistema de Estimativas da Emissão de Gases de Efeito Estufa (SEEG ,2019) é que, enquanto os setores considerados guardam certa relação com setores econômicos, os subsetores estão mais relacionados aos processos físicos, químicos e biológicos responsáveis pela emissão de GEE, o que facilita a sua interpretação. Por esta razão é interessante observarmos qual é a contribuição de cada setor e subsetor na emissão de GEE no Brasil. A tabela 1 mostra a contribuição de cada uma destas fontes para a emissão de GEE ocorrida no Brasil em 2018, medida em toneladas equivalente de dióxido de carbono [(t) $\left.\mathrm{CO}_{2} \mathrm{e}\right]$, avaliada pelo seu potencial de aquecimento global em 100 anos definido pelo 50 Relatório de Avaliação (GWP 100 AR5) proposto pelo IPCC (2014).

Observa-se na tabela 1 que o setor Mudança de Uso da Terra foi o que mais emitiu GEE no Brasil em 2018, com 42,65\% das emissões totais. O segundo maior setor é o da Agropecuária, com $26,35 \%$ das emissões, vindo depois o de Energia, com 21,04\%.

No que diz respeito aos subsetores, a maior emissão é proveniente das Alterações de Uso do Solo (40,11\%), seguida de Queima de Combustíveis (19,78\%) e Fermentação Entérica (16,29\%). É importante observar que as Alterações de Uso do Solo se constituem essencialmente nas queimadas relacionadas ao desmatamento, sendo a Fermentação Entérica a emissão de metano por ruminantes, especialmente bovinos. 
Tabela 1 - Setores e subsetores responsáveis pela emissão de gases de efeito estufa no Brasil em 2018.

\begin{tabular}{|c|c|c|c|c|}
\hline Setor & Subsetor & Valor & $\%$ Setor & $\%$ Total \\
\hline \multirow{7}{*}{$\begin{array}{l}\text { Agropecuária } \\
\text { (com calagem) }\end{array}$} & Cultivo do Arroz & 12.864 .897 & 2,52 & 0,66 \\
\hline & Fermentação Entérica & 315.899 .916 & 61,82 & 16,29 \\
\hline & Manejo de Dejetos Animais & 22.408 .290 & 4,38 & 1,16 \\
\hline & Queima de Resíduos Agrícolas & 5.165 .773 & 1,01 & 0,27 \\
\hline & Solos Agrícolas & 135.827.416 & 26,58 & 7,00 \\
\hline & Calagem & 18.869 .020 & 2,28 & 0,97 \\
\hline & Total do setor & 511.035 .313 & 100,00 & 26,35 \\
\hline \multirow{3}{*}{ Energia } & Fugitivas & 24.382 .260 & 5,98 & 1,26 \\
\hline & Queima de Combustíveis & 383.533 .837 & 94,02 & 19,78 \\
\hline & Total do setor & 407.916 .097 & 100,00 & 21,04 \\
\hline \multirow{7}{*}{$\begin{array}{l}\text { Processos } \\
\text { industriais }\end{array}$} & HFCs & 18.089.682 & 17,87 & 0,93 \\
\hline & Indústria Química & 3.734 .172 & 3,69 & 0,19 \\
\hline & Produção de Metais & 51.501 .698 & 50,87 & 2,66 \\
\hline & Produtos Minerais & 26.285 .347 & 25,96 & 1,36 \\
\hline & Uso de SF6 & 227.245 & 0,22 & 0,01 \\
\hline & $\begin{array}{l}\text { Uso Não-Energético de } \\
\text { Combustíveis e Uso de Solventes }\end{array}$ & 1.395 .767 & 1,38 & 0,07 \\
\hline & Total do setor & 101.233.912 & 100,00 & 5,22 \\
\hline \multirow{3}{*}{ Resíduos } & Tratamento de Efluentes Líquidos & 44.339 .171 & 48,25 & 2,29 \\
\hline & Resíduos Sólidos & 47.553 .664 & 51,75 & 2,45 \\
\hline & Total do setor & 91.892 .835 & 100,00 & 4,74 \\
\hline \multirow{3}{*}{$\begin{array}{l}\text { Mudança de Uso } \\
\text { da Terra (sem } \\
\text { calagem) }\end{array}$} & Alterações de Uso do Solo & 777.761 .771 & 94,04 & 40,11 \\
\hline & Resíduos Florestais & 49.281 .789 & 5,96 & 2,54 \\
\hline & Total das emissões do setor & 827.043 .560 & 100,00 & 42,65 \\
\hline \multicolumn{2}{|c|}{ Total das emissões (brutas) } & 1.939.121.718 & & 100,00 \\
\hline
\end{tabular}

Fonte: elaborado pelo autor com dados do SEEG (2018)

A proporção das emissões entre estes setores sofreu uma grande variação ao longo das últimas décadas, embora sem alterar a ordem dos setores mais importantes, como mostra a figura 1. Nessa figura observa-se que o Brasil atingiu um máximo de emissão de GEE em 2004. A partir deste ano ocorre uma diminuição da emissão que perdura até 2012, com um aumento em 2013 e, após, ligeiras quedas até 2018. Como pode ser observado na figura 1, o setor Mudança de Uso da Terra é o responsável por estas variações, na medida em que os demais apresentam um crescimento constante entre 1990 e 2014, apresentando ligeira queda até 2018. Como mostra 
a tabela 1, o subsetor Alterações de Uso do Solo representa 94,04\% das emissões do setor de Mudança de Uso da Terra.

Figura 1 - Emissão de gases de efeito estufa no Brasil entre 1990 e 2018, de acordo com o setor econômico

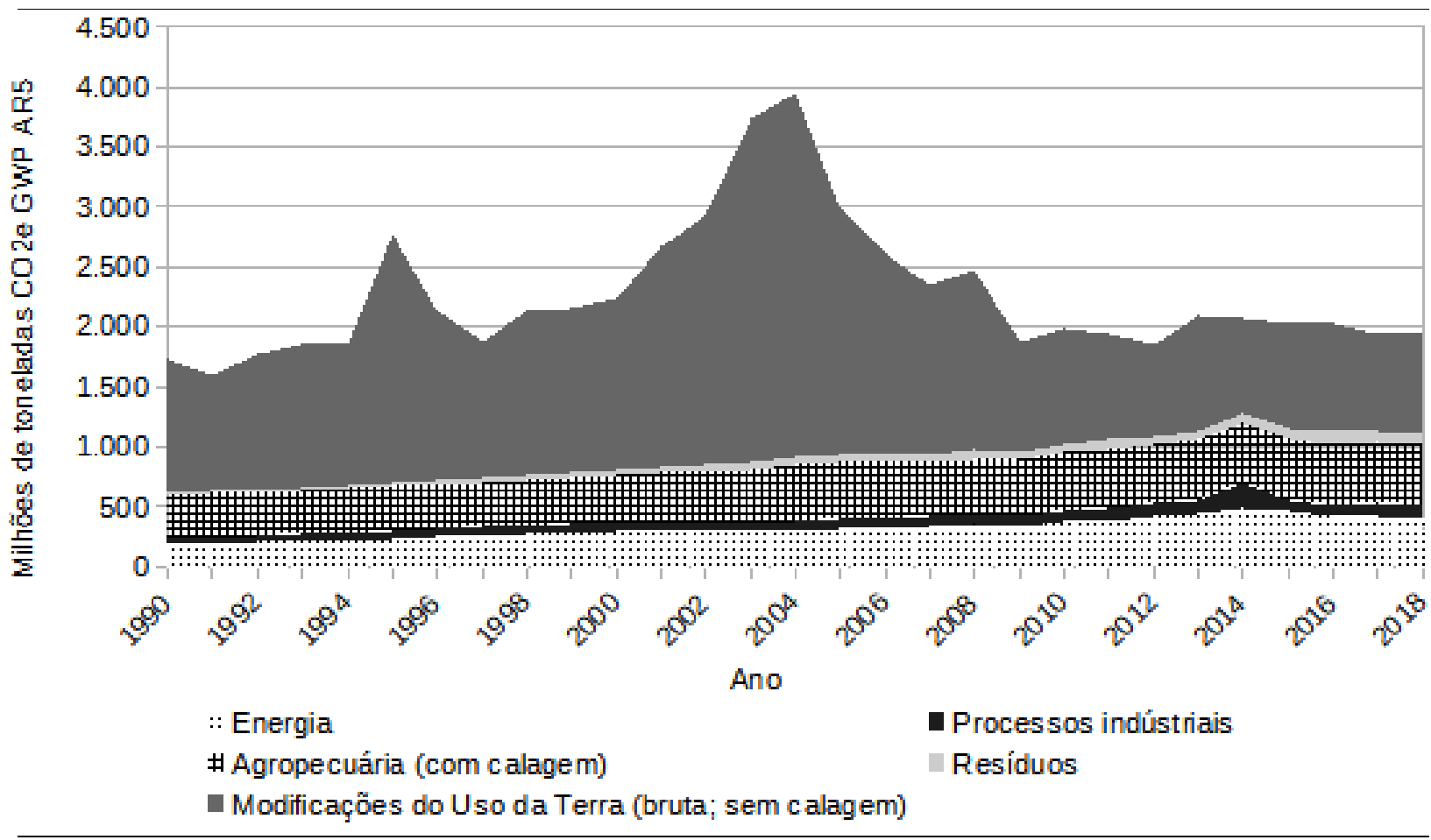

Fonte: elaborado pelo autor a partir de dados do SEEG (2019).

Como já comentado acima, a emissão por Alterações de Uso do Solo corresponde a das queimadas. Estes resultados, portanto, mostram que as queimadas são as principais responsáveis pela emissão de GEE no Brasil. A diminuição da emissão devido à Mudança de Uso da Terra, conforme mostra a figura 1, entre 2005 e 2012 devem-se, em grande parte, a uma série de políticas públicas destinadas ao combate ao desmatamento. Dentre estas políticas, destacam-se os Planos de Ação para a Prevenção e o Controle do Desmatamento da Amazônia e do Cerrado, implantados em 2004 e 2008, respectivamente (PLANOS DE PREVENÇÃO E CONTROLE DO DESMATAMENTO EM ÂMBITO FEDERAL, s.d.). Neste quadro, também merece destaque a Lei no 12.187 de 2.009, que instituiu a Política Nacional sobre Mudança do Clima (BRASIL, 2009). Porém, a partir de 2013 a área desmatada aumentou. Na Amazônia Legal ela passou de menos de 5.000 $\mathrm{km}^{2}$ em 2012 a cerca de $7.500 \mathrm{~km} 2$ em 2018. Embora no momento em que escrevemos este artigo ainda não havia dados oficiais para 2019 e 2020, é provável que o desmatamento no país cresceu significativamente neste período, como evidencia a drástica diminuição de recursos financeiros destinados a políticas relacionadas à mudança climática no Brasil (Tabela 2). 
Tabela 2 - Recursos financeiros destinados a políticas relacionadas à mudança climática

\begin{tabular}{lrr}
\hline Política & $2018(\mathrm{em} R \$)$ & 2019 (em R\$) \\
\hline Implementação da Política Nacional sobre Mudança Climática & $544.395,46$ & $22.496,34$ \\
Plano de Prevenção e Controle do Desmatamento & $175.897,39$ & $19.188,54$ \\
Política de Redução da Vulnerabilidade à Seca e à Mudança Climática & $460.493,17$ & 0,00 \\
Fundo para controle da Mudança Climática* & $6.368 .812,34$ & 0,00 \\
\hline
\end{tabular}

*Financiado majoritariamente por fundos internacionais

Fonte: Rittl; Angelo (2019)

\section{A Identidade de Kaya Composta}

A Identidade de Kaya foi desenvolvida pelo pesquisador japonês Yoichi Kaya que a apresentou ao Subgrupo Energia e Indústria do IPCC em 1990 (KAYA, 1990). A Identidade de Kaya permite uma análise interdisciplinar de fatores econômicos, demográficos e tecnológicos relacionados à emissão de GEE, de forma lógica e matematicamente rigorosa (IPCC, 2000). Neste sentido, ela permite relacionar a emissão de GEE com a produção de energia (E), a população (P) e o produto interno bruto (PIB), gerando coeficientes interessantes para avaliar as relações entre estas variáveis. A dedução da Identidade de Kaya tem como ponto de partida a identidade:

$$
\mathrm{CO} 2 e \equiv \mathrm{CO} 2 e
$$

a qual é dividida e multiplicada pela energia consumida $(E)$

ou seja,

$$
\text { CO2 } 2 e \equiv C O 2 e \frac{E}{E}
$$

$$
C 02 e \equiv \frac{C O 2 e}{E} E
$$

Efetuando a mesma operação com (PIB) e (P), se obtém a Identidade de Kaya, descrita como,

$$
C 02 e \equiv \frac{C O 2 e}{E} \frac{E}{P I B} \frac{P I B}{P} P
$$

No entanto, como visto na segunda seção, a emisssão de GEE não é provocada apenas pelo setor de energia. Isto torna menos eficaz a Identidade de Kaya usualmente empregada para a análise global das relações entre emissão de GEE e crescimento econômico. Por esta razão neste artigo as demais fontes de emissão de GEE foram adicionadas na identidade original, formando uma "Identidade de Kaya Composta" (IKC).

É importante observar que o setor Alterações de Uso do Solo, pelo menos no caso brasileiro, não possui uma relação direta com o PIB por habitante, como mostra a figura 2. Assim, por meio dos dados empregados para elaborar a figura 2 obteve-se um coeficiente de correlação 
negativo de $16 \%$ entre a emissão total e o PIB por habitante, e uma correlação positiva de $92,52 \%$ quando a emissão devido à Mudança de Uso da Terra foi subtraída da emissão total. Além disto, a partir das mesmas fontes de dados da figura 2 foi obtido um coeficiente de correlação negativo de 44,93\% entre a emissão por Mudança de Uso da Terra e o PIB por habitante. Esta correlação inversa entre desmatamento e crescimento econômico pode parecer paradoxal, na medida em que o grande argumento para o desmatamento é a necessidade de aumentar as áreas disponíveis para o desenvolvimento de atividades econômicas, especialmente agropecuárias.

Figura 2 - Emissões de GEE Total e por Mudança de Uso da Terra e PIB por habitante por habitante
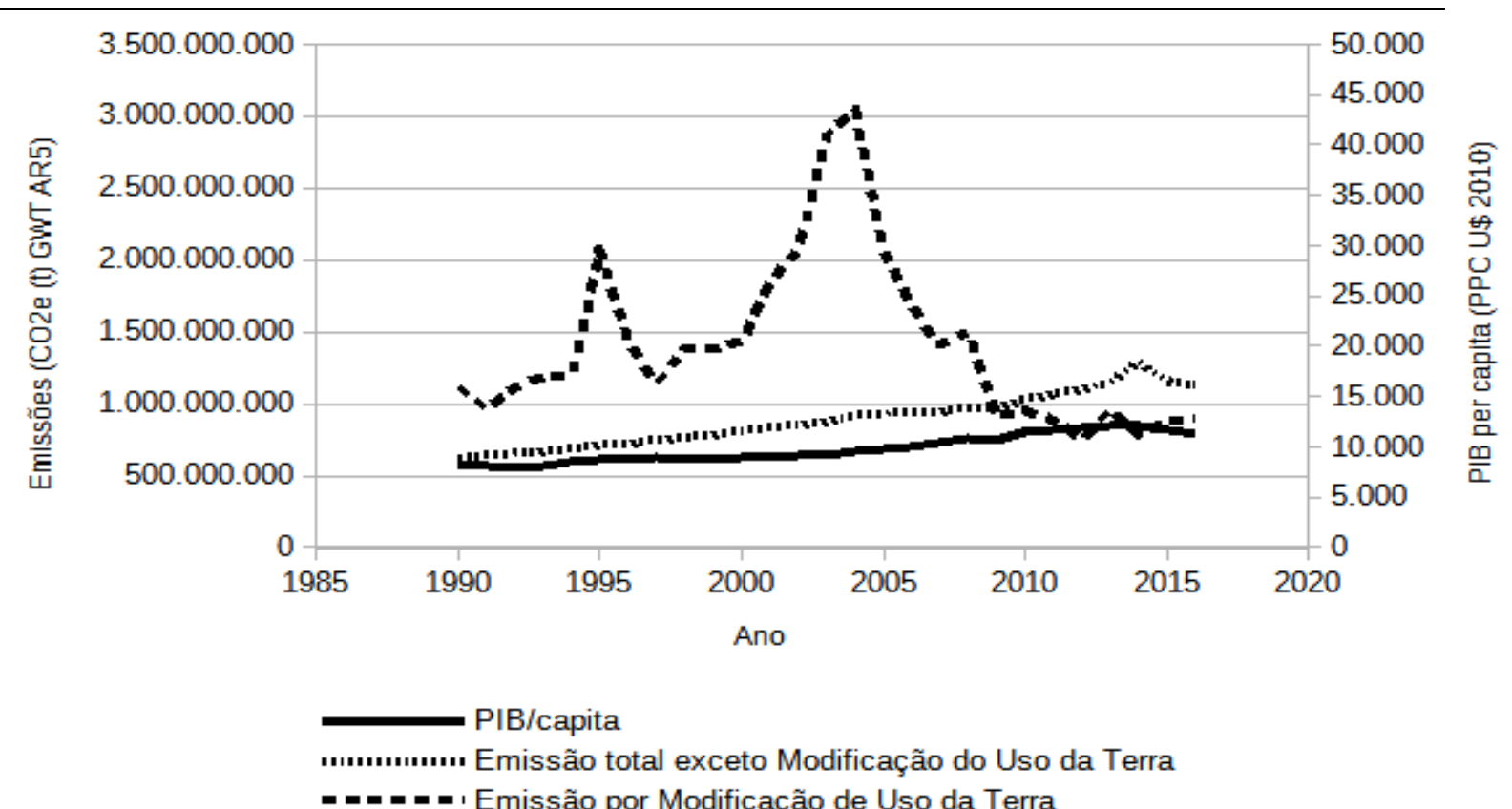

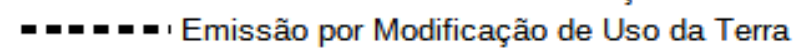

Fonte: elaborado pelo autor a partir de dados de emissões do SEEG (2019) e do PIB por habitante do World Bank Data (2020).

No entanto, a constatação feita neste artigo a partir dos dados que foram utilizados para a elaboração da figura 2 é corroborada por estudos macroeconômicos que detectaram uma pequena contribuição do desmatamento ao crescimento econômico da Amazônia (CARVALHO; MAGALHÃES; DOMINGUES, 2016).

A partir do mesmo procedimento descrito para a obtenção da identidade de Kaya (1990), a Identidade de Kaya Composta foi definida como,

$$
C O 2 e \equiv\left(\frac{C O 2 e E}{E} \frac{E}{P I B} \frac{P I B}{P} P\right)+\left(\frac{C O 2 e A+C O 2 e E+C O 2 e I+C O 2 e R}{P I B} \frac{P I B}{P} P\right)+C O 2 e M
$$

ou seja, 


$$
C 02 e \equiv\left(\frac{P I B}{P} P\right)\left[\left(\frac{C O 2 e(A+I+R)}{P I B}\right)+\left(\frac{C O 2{ }_{e} E}{E} \frac{E}{P I B}\right)\right]+C O 2 e M
$$

onde,

$\mathrm{CO} 2 \mathrm{e}\left(\right.$ ou $\left.\mathrm{CO}_{2} \mathrm{e}\right)=$ emissão de GEE em equivalente dióxido de carbono

$\mathrm{PIB}=$ produto interno bruto

$\mathrm{P}=$ população

$E=$ setor Energia

A =setor Agropecuária (com Calagem)

$\mathrm{I}=$ setor Processos Industriais

$\mathrm{R}=$ setor Resíduos

$\mathrm{M}=$ setor Mudança de Uso da Terra (sem Calagem)

Salientamos que, embora o subsetor Calagem seja considerado como um componente da Mudança de Uso da Terra pelo SEEG (2019), neste artigo ele foi retirado deste setor e incluído no setor Agropecuária. Isto porque a calagem é uma operação agrícola usualmente praticada, a qual possui uma relação direta com a produção agropecuária.

Na expressão (6), o PIB por habitante $\left(\frac{P I B}{P}\right)$ e a população $(P)$ são considerados variáveis de escala, ou seja, que determinam a magnitude da emissão por meio da sua influência sobre as demais variáveis. O valor do conteúdo de carbono da economia, excetuando o setor Mudança de Uso do Solo, depende do tipo de atividade desenvolvido em cada setor e das tecnologias nele empregadas. Na figura 3 é mostrado o conteúdo de carbono nos setores $(A+l+R)$ em seu conjunto, e para o setor de energia (E), assim como o PIB por habitante, com as suas linhas de tendência lineares, entre os anos de 1990 e 2018. Como mostra a figura 3, o conjunto dos setores ( $A+I+R)$ apresentou uma ligeira tendência a reduzir o seu conteúdo de carbono, sendo que o PIB por habitante cresceu. A partir dos dados empregados para a elaboração da figura 3 , foi calculada a taxa tendencial de diminuição do conteúdo de carbono dos setores $(A+I+R)$ de $0,73 \%$ ao ano, da qual resulta uma diminuição de $18,60 \%$ em todo o período.

No que diz respeito ao setor de energia, observa-se na figura 3 que o seu conteúdo de carbono apresentou uma ligeira alta que, calculada a partir da tendência linear, proporcionou como resultado uma taxa de crescimento de $0,34 \%$ ao ano, e de $9,89 \%$ no acumulado do período entre 1990 e 2018. No que diz respeito ao PIB por habitante, a partir da linha de tendência, foram obtidas as taxas de crescimento de $1,74 \%$ ao ano, e de $61,92 \%$ no acumulado do período.

De acordo com a expressão (6), o conteúdo de carbono do setor de energia é desmembrado em dois quocientes, $\frac{C O 2 e E}{E} \mathrm{e} \frac{E}{P I B}$. O quociente $\frac{C O 2 e E}{E}$ mostra o conteúdo de carbono do setor em unidades de energia, sendo o valor deste quociente definido pela composição da 
matriz energética do país. Já o quociente $\frac{E}{P I B}$ mostra a quantidade de energia por uma unidade de PIB, indicando a "intensidade energética" da economia. Este quociente relaciona-se ao tipo de atividade desenvolvida na economia e às práticas de conservação de energia. Vale salientar que a multiplicação dessas duas variáveis resulta na emissão de carbono equivalente por PIB do setor de energia.

Figura 3 - Conteúdo de carbono dos setores agropecuária (A), processos industriais (I) e resíduos (R), do setor de energia (E) e por habitante, de 1990 e 2018

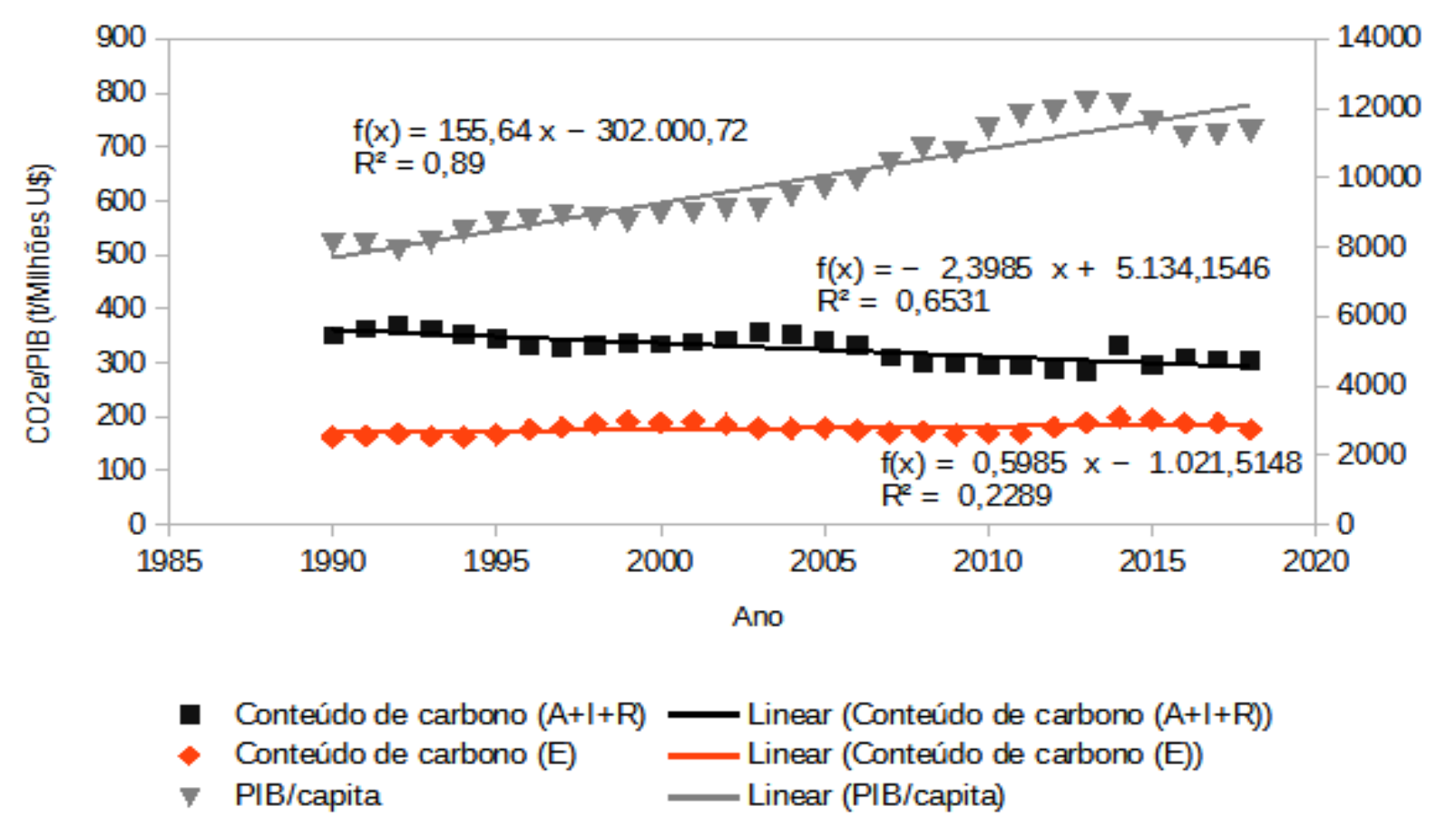

Fonte: elaborado pelo autor a partir de dados do SEEG (2019) e World Bank Data (2020)

Na figura 4 são mostrados o conteúdo de carbono da energia gerada no Brasil e a intensidade energética da economia brasileira no período de 1990 a 2018. Observa-se nesta figura que o conteúdo de carbono da energia e a intensidade energética da economia no Brasil são bastante estáveis. Tal estabilidade pode ser constatada pelos baixos índices de determinação das regressões lineares apresentadas na figura 4, abaixo. Além disto a partir dos dados empregados para elaborar a figura 4 obteve-se tendencialmente uma taxa média de crescimento geométrico entre 1990 e 2018 da intensidade energética da economia de 0,12\% ao ano, sendo 3,39\% no acumulado entre 1990 e 2018. 
Figura 4 - Intensidade energética da economia e conteúdo de carbono da energia no Brasil
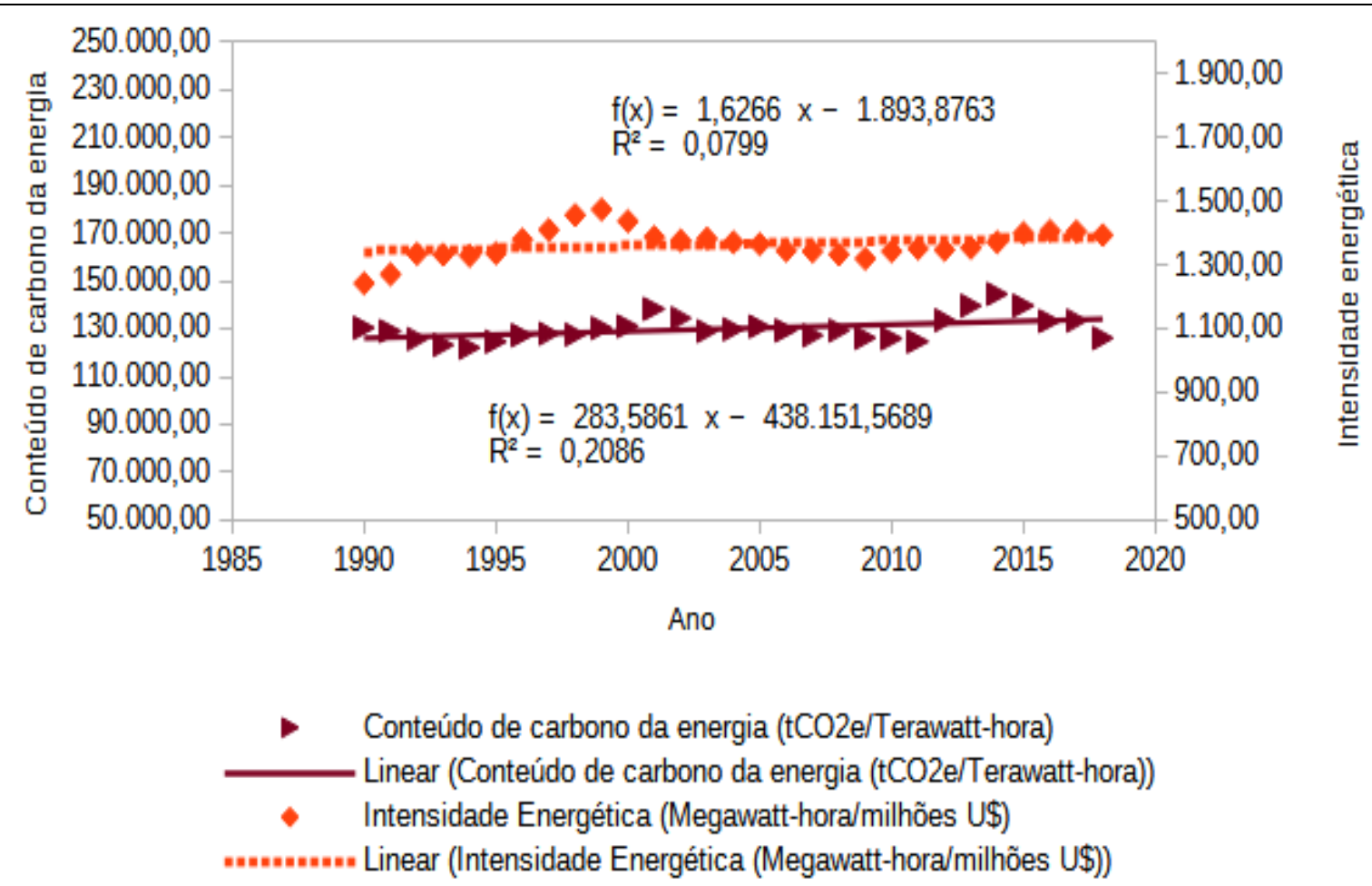

Fonte: elaborado pelo autor a partir de dados do SEEG (2019) e World Bank Data (2020)

Em relação ao conteúdo de carbono da energia, sua taxa média de crescimento geométrico é de $0,22 \%$ ao ano, sendo 6,29\% no acumulado entre 1990 e 2018 . Essas taxas, portanto, indicam uma baixa variação da intensidade energética da economia brasileira e do conteúdo de carbono da energia gerada no país entre 1990 e 2018.

\section{Definição dos cenários}

A Identidade de Kaya, como seu nome indica, é uma identidade matemática cujas propriedades possuem importantes implicações no seu uso para simulações. Ocorre que a Identidade de Kaya é uma construção lógico-matemática, não necessariamente expressando relações de causa e efeito. Neste sentido, a Identidade de Kaya não é adequada para a realização de previsões do comportamento dessas relações ao longo do tempo a partir de um estado inicial, na medida em que o comportamento das suas variáveis é mais complexo do que ela descreve (IPCC, 2000, p. 105).

Por outro lado, a Identidade de Kaya pode ser considerada como uma representação do estado de um sistema econômico em um momento específico. A partir deste pressuposto as simulações realizadas por meio da Identidade de Kaya fornecem as mudanças necessárias nas relações entre as variáveis, especialmente a relação entre o PIB por habitante e o conteúdo de 
carbono da economia no caso deste artigo, para que determinado cenário se concretize. É neste sentido que a Identidade de Kaya Composta aqui elaborada será empregada para a análise de cenários. Como base para a realização desses cenários foi considerado a taxa de crescimento geométrico médio da população brasileira de 0,35\% ao ano entre 2018 e 2050, de acordo com a projeção do IBGE (s.d.).

A definição da meta de redução de emissão de GEE foi realizada a partir das considerações contidas no 5을 Relatório de Avaliação do IPCC (IPCC, 2014). Neste relatório são elaborados vários cenários de emissão de GEE, os quais indicam que uma redução de 40 a $70 \%$ da emissão de origem antrópica até 2050 em relação à 2010, pode limitar o aquecimento global em 2ㅇ C em relação aos níveis observados anteriormente à era industrial (IPCC, 2014, p. 20). Tendo como base essas considerações, neste artigo os cenários foram elaborados para que se possa atingir uma redução de 50\% das emissões mundiais de GEE em 2050 em relação à 2010. Por outro lado, é importante salientar que os relatórios do IPCC contém muitos cenários de emissão, com resultados que variam significativamente de acordo com os pressupostos adotados para a sua elaboração, o que indica a grande complexidade de prever o efeito das atividades humanas sobre o aquecimento global. Considerando esses cenários, a redução do nível de emissão de GEE adotada neste artigo pode ser considerada bastante conservadora.

A emissão de GEE por habitante no Brasil em 2018 foram de 9,3 GWP (t) $\mathrm{CO}_{2} \mathrm{e}$, uma quantidade bem mais elevada do que a média mundial de 6,97 GWP (t) $\mathrm{CO}_{2} \mathrm{e}$ (SEEG, 2019). Isto implica que o país deveria realizar um esforço ainda maior do que a média mundial para que as metas estabelecidas pelo IPCC possam ser atingidas. Por outro lado, é importante observar que, retiradas as emissões geradas pelo desmatamento (Alterações de Uso dos Solos), as emissões de GEE no Brasil em 2018 se reduzem a 5,57 GWP (t) $\mathrm{CO}_{2}$ e por habitante, cerca de $20 \%$ inferior à média mundial. Esta inferioridade do nível de emissões por habitante no Brasil em relação à média mundial está relacionada ao fato do PIB por habitante brasileiro ser menor do que a média mundial em cerca de 7,5\% (WORLD BANK DATA, 2020).

No entanto, o fator mais importante responsável por esta diferença é o fato de a matriz energética brasileira ser composta por uma proporção maior de energias renováveis em relação à média mundial. De fato, enquanto a emissão média de GEE por unidade de energia no mundo é de 230.697 toneladas de $\mathrm{CO}_{2}$ e por Twh (Terawatts-hora), no Brasil esta média é de 141.305 toneladas de $\mathrm{CO}_{2} \mathrm{e}$ por Twh, (61,25\% da média mundial). Estes números podem ser explicados em maior detalhe examinando-se a composição relativa da matriz energética do Brasil em relação à da matriz energética mundial, mostrada na tabela 3. 
Tabela 2 - Matriz energética brasileira e mundial.

\begin{tabular}{|c|c|c|c|c|c|}
\hline Tipo & Fonte & Brasil & $\begin{array}{r}\text { Brasil } \\
(\mathrm{em} \%)\end{array}$ & Mundo & $\begin{array}{l}\text { Mundo } \\
\text { (em \%) }\end{array}$ \\
\hline \multirow{5}{*}{$\begin{array}{l}\text { Energias não } \\
\text { renováveis }\end{array}$} & Petróleo & $1.303,34$ & 40,28 & $53.181,12$ & 33,55 \\
\hline & Gás natural & 358,91 & 11,09 & $38.516,65$ & 24,30 \\
\hline & Carvão & 193,43 & 5,98 & $44.109,46$ & 27,82 \\
\hline & Nuclear & 38,96 & 1,20 & $6.711,46$ & 4,23 \\
\hline & Subtotal não renováveis & $1.894,63$ & 58,56 & $142.518,70$ & 89,90 \\
\hline \multirow{5}{*}{$\begin{array}{l}\text { Energias } \\
\text { Renováveis }\end{array}$} & Hidroelétrica & 966,74 & 29,88 & $10.367,54$ & 6,54 \\
\hline & Biocombustíveis & 245,03 & 7,57 & $1.040,05$ & 0,66 \\
\hline & Eólica & 120,48 & 3,72 & $3.156,85$ & 1,99 \\
\hline & Solar & 8,60 & 0,27 & $1.448,37$ & 0,91 \\
\hline & Subtotal renováveis & $1.340,85$ & 41,44 & $16.012,80$ & 10,10 \\
\hline \multirow[t]{2}{*}{ Baixo carbono } & Subtotal (renováveis + nuclear) & $1.379,81$ & 42,65 & $22.724,26$ & 14,33 \\
\hline & Total & $3.235,48$ & 100,00 & $158.531,50$ & 100,00 \\
\hline
\end{tabular}

Fonte: BP Statistical Rewiew (2019)

Observa-se na tabela 3 que as energias renováveis no Brasil (hidroelétrica, biocombustível, eólica e solar) compõem $41,44 \%$ da sua matriz energética. Adicionando a energia nuclear que, além das energias renováveis é considerada uma energia de baixo carbono (ALVIM et al., 2010), este percentual atinge $42,65 \%$ de fontes de energia de baixo carbono. Esta característica contrasta com a da matriz energética mundial, também mostrada na tabela 3 , que é composta por $10,1 \%$ de fontes de energia renováveis, e 14,33\% de fontes de energia de baixo carbono, quando adicionamos a energia nuclear. Portanto, o abastecimento de energia no mundo é muito mais dependente do petróleo, do gás e, principalmente, do carvão mineral, do que no Brasil, sendo estas as fontes que mais emitem GEE por unidade de energia.

Os dados mostrados na tabela 3 se refletem no conteúdo de carbono do setor de energia, o qual, em 2018, no Brasil foi de 1,414 $\mathrm{tCO}_{2} \mathrm{e}$ por tep (tonelada equivalente de petróleo), enquanto nos países da OCDE foi de $2,2 \mathrm{tCO}_{2}$ e por tep, sendo que a média mundial foi de 2,33 $\mathrm{tCO}_{2} \mathrm{e}$ por tep (RESENHA ENERGÉTICA BRASILEIRA, 2019). De qualquer forma, a elevada média de emissão de GEE por habitante no Brasil em relação à média mundial implica que as emissões de GEE do Brasil em 2018 deveriam ser divididas por cerca de 2,54, devendo ser reduzidas de 1.939.121.718 para 762.899.003 GWP $\left(\mathrm{t}\right.$ ) $\mathrm{CO}_{2} \mathrm{e}$, para que o país contribua proporcionalmente com os demais para diminuir pela metade a emissão de GEE no mundo até 2050 em relação ao ano de 2010, meta proposta pelo IPCC (2014).

Os cenários de crescimento econômico foram elaborados para fornecer subsídios à discussão de qual PIB por habitante pode ser considerado compatível com esta meta estabelecida pelo IPCC (2014). Em todos os cenários foi definida uma taxa anual de diminuição do 
desmatamento de forma que este seja eliminado (desmatamento zero) até 2050, restando apenas no setor Mudança de Uso da Terra (exceto Calagem), a emissão de GEE relativa aos Resíduos Florestais. Assim, os cenários foram constituídos para indicar qual seria o conteúdo de carbono da economia compatível com taxas de crescimento do PIB por habitante de 0\% (sem crescimento), 1\%, $2 \%$ e $3 \%$ ao ano, constituindo os cenários 1, 2, 3 e 4, respectivamente.

O conteúdo de carbono na economia foi definido fixando a mesma taxa de variação para o conjunto formado pelos setores Agropecuária, Processos Industriais e Resíduos $(A+I+R)$ e, separadamente, para o setor de energia (E). Para isto optou-se por manter o valor inicial da intensidade energética da economia e variar apenas o conteúdo de carbono por unidade de energia. Este procedimento se justifica pelo fato do conteúdo de carbono da energia no Brasil apresentar uma tendência de crescimento maior do que a intensidade energética da economia (embora estatisticamente ambas podem ser consideradas estáveis), conforme mostrado na figura 4, apresentada na segunda seção.

No entanto, salientamos que a variação do conteúdo de carbono do setor energético é resultante da multiplicação da variação dos dois quocientes relativos à energia. Como foi considerada uma variação nula da intensidade energética da economia $\left(\frac{E}{P I B}\right)$, a variação do conteúdo de carbono do setor de energia $\left(\frac{\mathrm{CO}_{2} e E}{E}\right)$ corresponde à variação do conteúdo de carbono da economia.

\section{Resultados das simulações}

Observa-se na tabela 4, de acordo com o cenário 1 (sem crescimento anual do PIB/hab.), que apenas para manter o PIB por habitante de 2018, o conteúdo de carbono dos setores (A+I+R) da economia em 2050 deverá ser de 57,44\% do observado em 2018. Nessa mesma tabela observa-se, também, que esta diminuição implica em dividir o conteúdo de carbono desses setores por 1,74 entre 2018 e 2050, a uma taxa geométrica negativa de 1,72\% ao ano. Como também pode ser observado na tabela 4 , foi considerado que a sua intensidade energética da economia não se alteraria, do que resulta que a taxa de diminuição do conteúdo de carbono da energia seria equivalente ao dos setores $(A+I+R)$. Os demais resultados dessas simulações dos cenários 1, 2, 3 e 4 elaborados são mostrados na tabela 4. 
Tabela 4 - Taxas de diminuição do conteúdo de carbono de acordo com diferentes taxas de crescimento do PIB/habitante

\begin{tabular}{|c|c|c|c|c|c|c|}
\hline Variável & Parâmetro & $\begin{array}{r}\text { Condições } \\
\text { iniciais (2018) }\end{array}$ & $\begin{array}{r}\text { Sem } \\
\text { crescimento } \\
\text { do } \mathrm{PIB} / \text { hab. }\end{array}$ & $\begin{array}{r}\text { Crescimento } \\
\text { do PIB/hab. } \\
\text { de } 1 \% \text { a.a. }\end{array}$ & $\begin{array}{r}\text { Crescimento } \\
\text { do PIB/hab. } \\
\text { de } 2 \% \text { a.a. }\end{array}$ & $\begin{array}{r}\text { Crescimento } \\
\text { do PIB/hab. } \\
\text { de } 3 \% \text { a.a. }\end{array}$ \\
\hline \multirow{4}{*}{$\begin{array}{l}\text { PIB per capita } \\
\text { (U\$/hab.) }\end{array}$} & Valor & 11.131 & 11.131 & 15.305 & 20.978 & 28.665 \\
\hline & $\begin{array}{l}\text { Taxa anual de } \\
\text { crescimento (\%) }\end{array}$ & & 0,00 & 1,00 & 2,00 & 3,00 \\
\hline & 2050/2018 (\%) & & 0,00 & 37,49 & 88,45 & 157,51 \\
\hline & $2018 / 2050$ & & 1,00 & 0,73 & 0,53 & 0,39 \\
\hline \multirow{4}{*}{$\begin{array}{l}\text { Conteúdo de carbono } \\
\text { de }(\mathrm{A}+\mathrm{I}+\mathrm{R})^{*} \\
\left(\mathrm{tCO} \mathrm{C}_{2} \mathrm{e} / \text { milhões U\$) }\right.\end{array}$} & Valor & 303,41 & 174,27 & 126,75 & 92,47 & 67,67 \\
\hline & $\begin{array}{l}\text { Taxa anual de } \\
\text { crescimento (\%) }\end{array}$ & & $-1,72$ & $-2,69$ & $-3,64$ & $-4,58$ \\
\hline & $2050 / 2018(\%)$ & & 57,44 & 41,77 & 30,48 & 22,31 \\
\hline & $2018 / 2050$ & & 1,74 & 2,39 & 3,28 & 4,48 \\
\hline \multirow{4}{*}{$\begin{array}{l}\text { Conteúdo de carbono } \\
\text { por unidade de } \\
\text { energia } \\
\left.\text { (tCO } \mathrm{tC}_{2} \mathrm{e} / \mathrm{tep}\right)\end{array}$} & $\underline{\text { Valor }}$ & 1,414 & 0,812 & 0,591 & 0,431 & 0,315 \\
\hline & $\begin{array}{l}\text { Taxa anual de } \\
\text { crescimento (\%) }\end{array}$ & & $-1,72$ & $-2,69$ & $-3,64$ & $-4,58$ \\
\hline & $2050 / 2018(\%)$ & & 57,44 & 41,77 & 30,48 & 22,31 \\
\hline & $2018 / 2050$ & & 1,74 & 2,39 & 3,28 & 4,48 \\
\hline \multirow{4}{*}{$\begin{array}{l}\text { Intensidade } \\
\text { energética da } \\
\text { economia } \\
\text { (tep/Milhões U\$) }\end{array}$} & Valor & 124,26 & 124,26 & 124,26 & 124,26 & 124,26 \\
\hline & $\begin{array}{l}\text { Taxa anual de } \\
\text { crescimento (\%) }\end{array}$ & & 0,00 & 0,00 & 0,00 & 0,00 \\
\hline & $2050 / 2018(\%)$ & & 100,00 & 100,00 & 100,00 & 100,00 \\
\hline & $2018 / 2050$ & & 1,00 & 1,00 & 1,00 & 1,00 \\
\hline \multirow{4}{*}{$\begin{array}{l}\text { Emissões pela } \\
\text { Mudança de Uso da } \\
\text { Terra (exceto a } \\
\text { Calagem) } \\
\left(\mathrm{tCO}_{2} \mathrm{e}\right)\end{array}$} & Valor & 827.043 .560 & 49.281 .789 & 49.281 .789 & 49.281 .789 & 49.281 .789 \\
\hline & $\begin{array}{l}\text { Taxa anual de } \\
\text { crescimento (\%) }\end{array}$ & & $-8,44$ & $-8,44$ & $-8,44$ & $-8,44$ \\
\hline & $2050 / 2018(\%)$ & & 5,96 & 5,96 & 5,96 & 5,96 \\
\hline & $2018 / 2050$ & & 16,78 & 16,78 & 16,78 & 16,78 \\
\hline
\end{tabular}

* sem Modificação do Uso da Terra exceto a calagem

Fonte: simulações elaboradas pelo autor a partir de dados do IBGE (2020), World Bank Data (2020) e SEEG (2019)

Os resultados obtidos com o primeiro cenário, sem crescimento do $\mathrm{PIB} /$ habitante portanto, indicam a necessidade de uma forte inflexão da tendência do conteúdo de carbono da economia brasileira. Como já mencionado, a partir dos dados empregados para elaborar a figura 3, apresentada na terceira seção, entre 1990 e 2018 foi constatada uma tendência de diminuição de $0,73 \%$ ao ano do conteúdo de carbono dos setores $(A+I+R)$, enquanto o conteúdo de carbono da energia apresenta uma tendência a apresentar uma taxa de crescimento positiva de $0,34 \%$ ao ano.

É importante salientar que esta necessidade de reduzir o conteúdo de carbono da economia seria muito maior caso a taxa de redução da emissão de GEE devido ao desmatamento de $8,44 \%$ ao ano não seja atingida, como mostram as simulações apresentadas na tabela 4. Para situar a ordem de grandeza deste ritmo de diminuição do desmatamento, foi calculada a taxa de 
redução da emissão provocada pela Mudança de Uso da Terra entre 2004 e 2012, período em que o desmatamento foi reduzido com maior intensidade no Brasil, como discutido na segunda seção. O resultado obtido foi uma taxa geométrica negativa anual de 5,18\% ao ano, da qual resulta que as emissões em 2012 correspondiam a 24,31\% das emissões de 2004. Portanto, para reduzir as emissões de GEE em uma quantidade compatível com uma diminuição de $50 \%$ das emissões mundiais em 2050 (em relação à 2010), o combate ao desmatamento no Brasil deveria ser ainda mais intenso do que no período em que ele foi mais efetivo. No entanto, o que se observa atualmente é justamente o contrário, conforme discutido na segunda seção.

Os resultados obtidos com o primeiro cenário, portanto, indicam uma grande dificuldade para o cumprimento das metas de controle da emissão de GEE no Brasil, de acordo com os níveis definidos pelo IPCC, mesmo com um crescimento econômico apenas suficiente para manter o PIB por habitante observado em 2018. Esses resultados tornam previsível que esta dificuldade será ainda maior no caso de haver um crescimento não nulo do PIB por habitante no Brasil, o que pode ser constatado observando-se os resultados relativos ao cenário 2, mostrados na tabela 4 . Neste cenário, o crescimento do PIB por habitante no Brasil a uma taxa de $1 \%$ ao ano a partir de 2018 faria com que o país em 2050 atingisse um PIB por habitante de U\$ 15.305 (em paridade de poder de compra), equivalente aproximadamente ao do Irã em 2016, porém inferior ao da Argentina ou ao do Uruguay em 2016 (WORLD BANK DATA, 2020). Conforme a tabela 4, no segundo cenário o conteúdo de carbono da economia brasileira (excetuando o setor Mudança de Uso da Terra) deveria ser reduzido em 2,52 vezes entre 2018 e 2050, o que resulta em uma taxa geométrica média negativa de $2,85 \%$ ao ano.

Observa-se na tabela 4 que a diminuição do conteúdo de carbono prevista no cenário 3 (2\% de crescimento anual do PIB por habitante) é de $3,8 \%$ ao ano, mais do que o dobro da observada no cenário 1 e um terço maior do que a do cenário 2 (crescimento anual do PIB/hab. de $1 \%$ a.a.). Isto implicaria em uma diminuição de 3,45 vezes do conteúdo de carbono da economia em 2050 em relação à 2018. Vale observar que o crescimento do PIB por habitante no Brasil a uma taxa de $2 \%$ ao ano a partir de 2018 (cenário 3) faria com que o país em 2050 atingisse um PIB por habitante de U\$20.978 (em paridade de poder de compra), valor um pouco superior ao PIB por habitante da Argentina ou do Uruguay em 2016 (WORLD BANK DATA, 2020).

No que diz respeito ao cenário 4 (crescimento anual do PIB/habitante de $3 \%$ a.a.), observa-se na tabela 4 que o conteúdo de carbono da economia deveria ser reduzido em 4,72 vezes entre 2050 e 2018. Neste caso, em 2050 ele corresponderia a 21,18\% do valor de 2018, o que representa uma taxa geométrica média negativa de $4,73 \%$ ao ano. É interessante observar 
que um crescimento do PIB por habitante no Brasil a uma taxa de $3 \%$ ao ano a partir de 2018 faria com que o país em 2050 atingisse U\$28.665 por pessoa (em paridade de poder de compra), valor um pouco superior ao de Portugal em 2016 (WORLD BANK DATA, 2020).

Considerando a fraca tendência de diminuição do conteúdo de carbono da economia brasileira observada entre 1990 e 2018, como mostrado na figura 3 apresentada na terceira seção, as diminuições do conteúdo de carbono mostradas nos cenários 3 e 4 podem ser consideradas muito difíceis de serem alcançadas. Neste sentido é interessante realizarmos, mesmo que brevemente, algumas considerações sobre as possibilidades tecnológicas existentes para a diminuição das emissões de GEE por alguns dos subsetores mostrados na tabela 1 (apresentada na segunda seção). Conforme a tabela 1, os subsetores Queima de Combustíveis e Fermentação Entérica provocaram 19,78\% e 16,29\% das emissões de GEE, respectivamente, o que representa $36,07 \%$ do total das emissões de 2018. Em termos absolutos, estes dois subsetores em seu conjunto provocaram 699.433.753 GWP (t) $\mathrm{CO}_{2}$ e de emissão de GEE.

A Queima de Combustíveis, conforme mostrado na tabela 1, provocou a emissão de GEE de 383.533.313 GWP (t) $\mathrm{CO}_{2} \mathrm{e}$. Neste caso seria necessário incentivar fortemente a substituição do petróleo por fontes de energia renováveis. A título de comparação, os países da Organização de Cooperação e Desenvolvimento (OCDE), entre 1973 e 2018 (45 anos), promoveram uma redução de $17,2 \%$ do petróleo e derivados na sua matriz energética (o que representa uma taxa geométrica média negativa de 1,21\% ao ano no período). Esta redução reflete um grande esforço de substituição desses produtos, decorrente principalmente dos choques nos preços de petróleo ocorridos em 1973 (ano em que o preço passou de US\$ 3 para US\$ 12 por barril), em 1979 (de US\$ 12 para US\$ 40), e a partir de 1998, quando teve início um novo ciclo de aumentos.

A partir de 2016, observa-se certa retração nos preços de petróleo (RESENHA ENERGÉTICA BRASILEIRA, 2019). A partir de dados publicados pela British Petroleum (BP STATISTICAL REVIEW, 2020), calculamos que nos últimos 29 anos (1990 a 2019) a participação do petróleo e derivados na matriz energética brasileira se manteve praticamente estável (aumento de 0,75\% em todo o período), apesar de um aumento de 8,19\% da participação das fontes de energia eólica e solar, o qual, no entanto, foi compensado por uma diminuição de $8,94 \%$ da participação da energia hidroelétrica no período.

Uma alteração da matriz energética brasileira para uma redução da emissão de GEE compatível com as metas definidas pelo IPCC (2014), portanto, implicaria em uma forte alteração da tendência observada nas últimas décadas. Além disto, é difícil conceber como o Brasil poderia diminuir a intensidade energética da sua economia. Isto porque, o crescimento do PIB por 
habitante implica em um aumento da produtividade global do trabalho, a qual é realizada essencialmente pela substituição da energia humana por outras fontes de energia.

No que diz respeito a Fermentação Entérica, de acordo com a tabela 1, ela provocou a emissão de 315.899.916 GWP (t) $\mathrm{CO}_{2}$ e em 2018, a qual decorre do gás metano emitido pelos ruminantes. A emissão de gás metano pelos bovinos pode ser reduzida por ajustes na alimentação e pelo aumento da produtividade animal (na medida em que esta representa mais produção por quantidade de alimento ingerido). No entanto, a possibilidade de tal redução é, no máximo, da ordem de 20\% (BERCHIELLI et al., 2012). Considerando uma contribuição equitativa entre os setores, isto torna a redução da Fermentação Entérica por meio da aplicação de tecnologias de produção insuficiente diante da necessidade de reduzi-la em 45,45\%, 60,33\%, $71,06 \%$ ou $78,82 \%$ para assegurar um crescimento do PIB por habitante de $0 \%, 1 \%, 2 \%$ ou $3 \%$ ao ano, respectivamente, entre 2018 e 2050.

Assim, a principal forma de reduzir a liberação de GEE pela Fermentação Entérica seria a diminuição do número de ruminantes domésticos no Brasil, dos quais o rebanho bovino possui atualmente 213.523 .056 cabeças (maior do que a população humana, de 208.494.900), o bubalino, 1.390 .066 cabeças, e os caprinos e ovinos, em conjunto, 29.645 .598 cabeças. Como o rebanho bovino é muito maior do que os demais, a sua diminuição seria imprescindível para uma redução significativa da emissão de GEE por Fermentação Entérica.

Embora a diminuição do valor econômico decorrente da diminuição do rebanho bovino possa ser, em parte, compensada pelo aumento da produtividade (que, como vimos, contribui para reduzir a emissão de GEE por unidade animal), é provável que ela dificultaria o crescimento econômico. É interessante observar que este aumento de produtividade poderia ser obtido pela recuperação de pastagens degradadas, processo que implica em uma fixação de dióxido de carbono que em parte compensaria a emissão de GEE provocada pelos ruminantes.

Além disso, a substituição das terras liberadas pela pecuária de ruminantes pelo reflorestamento, o qual se constitui em uma importante forma de fixação de dióxido de carbono, poderia compensar, pelo menos em parte, as perdas econômicas decorrentes da diminuição do rebanho. Neste sentido seria necessário evitar que as terras liberadas pela pecuária bovina fossem ocupadas por culturas anuais que, por serem mais mecanizadas, consomem quantidades maiores de combustíveis fósseis, cuja emissão de GEE, provavelmente, seria mais elevada do que a provocada pelos ruminantes.

Enfim, é importante observar que o Brasil, pela sua densidade demográfica relativamente baixa, possui amplas possibilidades de promover o sequestro de dióxido de carbono da atmosfera 
(denominada genericamente de medidas de "mitigação" das emissões) por meio da recuperação de áreas desmatadas, especialmente da Mata Atlântica, mas também em outros biomas como o Cerrado, a Caatinga e a Amazônia. A criação de Áreas Protegidas ou de Conservação, assim, pode desempenhar um papel importante para que o Brasil possa atingir as metas estabelecidas pelo IPCC (2014).

Além disso, várias medidas de mitigação relacionadas à agricultura são possíveis de serem implementadas. Merece destaque neste sentido, o Plano Setorial de Mitigação e de Adaptação às Mudanças Climáticas para a Consolidação de uma Economia de Baixa Emissão de Carbono na Agricultura (Plano ABC), do Ministério da Agricultura e Abastecimento (MAPA, 2012). Este plano previa uma série de medidas para a diminuição direta das emissões e, principalmente, da sua compensação por meio da fixação de dióxido de carbono, de um total estimado entre 133,9 e 162,9 milhões de $\mathrm{tCO}_{2} \mathrm{e}$ até 2020, conforme mostrado na tabela 5.

Tabela 3 - Processos Tecnológicos, compromisso nacional relativo (aumento da área de adoção ou uso) e potencial de mitigação por redução de GEE do Plano ABC.

\begin{tabular}{lcc}
\hline Processo Tecnológico & $\begin{array}{c}\text { Compromisso até } 2020 \text { (aumento } \\
\text { de milhões de hectares ou m }{ }^{3} \text { ) }\end{array}$ & $\begin{array}{c}\text { Potencial de mitigação } \\
\left.\text { (em milhões de } \mathrm{CO}_{2} \mathrm{e}\right)\end{array}$ \\
\hline Recuperação de pastagens degradadas & 15,0 & 83 a 104 \\
Integração Lavoura- Pecuária-Floresta & 4,0 & 18 a 22 \\
Sistema Plantio Direto & 8,0 & 16 a 20 \\
Fixação Biológica de Nitrogênio $\left(\mathrm{m}^{3}\right)$ & 5,5 & 10 \\
Florestas Plantadas* & 3,0 & --- \\
Tratamento de Dejetos Animais $\left(\mathrm{m}^{3}\right)$ & 4,4 & 6,9 \\
\hline Total & --- & 133,9 a 162,9 \\
\hline
\end{tabular}

* não computado o potencial de mitigação da emissão de GEE

Fonte: adaptado a partir de MAPA (2012, p. 20)

É interessante observar que a mitigação das emissões de GEE proposta no Plano ABC seria mais do que o dobro das emissões de GEE do subsetor Resíduos Florestais (49.281.789 $\mathrm{tCO}_{2} \mathrm{e}$, como mostrado na tabela 4). Por outro lado, observa-se que o Plano ABC não possui medidas em prol de sistemas alternativos, como os orgânicos e agroecológicos, que permitiriam reduzir a emissão de GEE pela agricultura por meio da diminuição da mecanização e do uso de agrotóxicos e outros insumos químicos, cuja intensa utilização é uma característica marcante da agricultura brasileira, especialmente de culturas como a soja e o milho, que ocupam a maior área plantada no Brasil. 
Em síntese, os resultados mostrados anteriormente na tabela 4 indicam que, mesmo considerando todas as medidas de mitigação discutidas nos parágrafos anteriores, as condições necessárias para que o Brasil possa conciliar crescimento econômico e diminuição da emissão de GEE de forma a contribuir efetivamente para amenizar os impactos da mudança climática, podem ser consideradas muito difíceis de serem asseguradas.

\section{Considerações finais}

Os resultados obtidos neste artigo permitem duas conclusões principais:

1) uma drástica diminuição do desmatamento é praticamente imprescindível para que o Brasil possa efetivamente limitar as suas emissões de GEE;

2) a diminuição do conteúdo de carbono da economia brasileira necessária para que o Brasil possa contribuir de forma equitativa com os demais países para que as metas estabelecidas pelo IPCC (2014) sejam atingidas representa uma severa limitação ao crescimento do PIB por habitante do país, mesmo a taxas modestas.

Reforça estas conclusões o crescente consenso de que a limitação do aumento da temperatura da atmosfera em até $2 \stackrel{\circ}{ } \mathrm{C}$ implica em um risco elevado de eventos climáticos extremamente prejudiciais as sociedades humanas e aos ecossistemas naturais. Por esta razão um relatório do IPCC publicado em 2019 propõe que o limite de aumento da temperatura da atmosfera seja diminuído para 1,5 으, o que implica em reduções muito mais severas da emissão de GEE do que as consideradas neste artigo.

Enfim, é interessante observar que os resultados obtidos neste artigo são, pelo menos aparentemente, contraditórios com a concepção de que medidas para a limitação da emissão de GEE possam ser conciliadas com altas taxas de crescimento econômico. Baseiam-se nesta concepção o "Green New Deal" (Novo Pacto Verde) proposto durante a campanha do candidato Bernie Sanders a presidência dos EUA (SANDERS, s.d.), o qual é inspirado nas políticas anticíclicas do governo de Franklin Delano Roosevelt durante a Depressão de 1929, mas desta vez baseandose na promoção de uma economia "de baixo carbono". Outro grande programa de promoção do crescimento econômico a partir da luta contra a emissão de GEE é o "Green Deal" que tem sido discutido pela União Europeia (GADREY, 2020). Tais propostas pretendem estimular o crescimento econômico por meio de vultosos investimentos, da ordem de 16 trilhões de dólares nos EUA (SANDERS, s.d.) e de um trilhão de euros, no caso da União Europeia (EUROPE UNION, s.d.). 
Tais investimentos permitiriam desencadear ações como o desenvolvimento de fontes de energia renováveis e de conservação de energia que, além de serem medidas contra o aquecimento global, provocariam uma massiva criação de empregos. No entanto, os Estados Unidos e a União Europeia estão entre os países com os maiores PIB por habitante do mundo, e com matrizes de energia altamente dependentes de combustíveis fósseis. A emissão de GEE por esses países (ao lado da China) são as que mais contribuem para o aquecimento global (WORLD BANK DATA, 2020).

Embora a análise desta questão esteja além dos objetivos deste artigo, os resultados nele apresentados suscitam uma grande incerteza sobre a real capacidade desses países em assegurar, ao mesmo tempo, altas taxas de crescimento econômico com uma diminuição do conteúdo de carbono das suas economias compatíveis com as metas estabelecidas pelo IPCC (2014). Neste sentido, os resultados apresentados neste artigo indicam que a necessidade de limitar a emissão de GEE devido as mudanças climáticas a ele associadas se constitui em um sério obstáculo ao crescimento econômico mundial, não apenas no Brasil. A magnitude deste obstáculo, no entanto, tem sido largamente negligenciado pelas sociedade contemporâneas.

\section{Referências}

ALVIM, C. F.; FERREIRA, O. C.; GUIDICI, O. M.; EIDELMAN, F., FERREIRA, P. A. F.; BERNARDES, M. A. S. Comparação da emissão de gases de efeito estufa (GEE) na geração nuclear de eletricidade no Brasil com as de outras fontes. Economia e Energia, Rio de Janeiro, no 79, p. 3-20, 2010.

ARAÚJO, R. et. al., Territórios e alianças políticas do pós-ambientalismo. Estudos Avançados, São Paulo vol. 33, no 95, p. 67-90, jan./abr. 2019.

ARTAXO, P. Mudanças Climáticas e o Brasil. REVISTA USP, São Paulo, n. 103, p. 8-12, 2014.

BP STATISTICAL REVIEW OF WORLD ENERGY. British Petroleum, 2020 Disponível em:

www.bp.com/statisticalreview ; acesso em: 10 de setembro de 2020.

BERCHIELLI, T. T.; MESSANA, J. D.; CANESIN, R. C. Produção de metano entérico em pastagens tropicais. Rev. Bras. Saúde Prod. Anim., Salvador, v. 13, no 4, p. 954-968, 2012.

BRASIL, Lei no 12.187 de 2.009, Institui a Política Nacional sobre Mudança do Clima. Diário Oficial da União - Edição Extra de 29/12/2009, (p. 109, col. 2).

CARVALHO, T. S.; MAGALHÃES, A. S.; DOMINGUES, E. P. Desmatamento e a contribuição econômica da floresta na Amazônia. Est. Econ., São Paulo, vol. 46, p. 499-531, abr.-jun. 2016.

EUROPE UNION, s.d. EU climate action and the European Green Deal, s.d. Disponível em: https://ec.europa.eu/clima/policies/eu-climate-action_en, acesso em: 12/09/2020).

FEIJÓ, G. dos R.; RANGEL, J. J. de A. Análise do comportamento das emissões de dióxido de carbono $\left(\mathrm{CO}_{2}\right)$ do Brasil e de outros países por meio da Identidade de Kaya e do Perfil de Emissões. Desenvolvimento e Meio Ambiente, vol. 46, p. 1-22, 2018. 
GADREY, J. Le "Green New Deal” aux États Unis et en Europe. Les Possibles, Paris, no 23, p. 100110, 2-020.

IBGE. Projeção da população do Brasil e das Unidades da Federação, s.d. (disponível em: https://www.ibge.gov.br/apps/populacao/projecao/, acesso em: 12/092020)

IPCC. Climate Change. The IPCC Response Strategies. Response Strategies Working Group. World Meteorological Organization/United Nations Environment Program, 1990.

IPCC. Emissions Scenarios. Cambridge: Cambridge University Press, 2000. Disponível em https://www.ipcc.ch/report/emissions-scenarios/, acesso: 12/09/2020.

IPCC. Climate Change 2014: Synthesis Report. Contribution of Working Groups I, II and III to the Fifth Assessment Report of the Intergovernmental Panel on Climate Change [Core Writing Team, R.K. Pachauri and L.A. Meyer (eds.)]. IPCC, Geneva, Switzerland, 2014.

IPCC. Global Warming of $1.5^{\circ} \mathrm{C}$. An IPCC Special Report on the impacts of global warming of $1.5^{\circ} \mathrm{C}$ above pre-industrial levels and related global greenhouse gas emission pathways, in the context of strengthening the global response to the threat of climate change, sustainable development, and efforts to eradicate poverty [Masson-Delmotte, V., P. Zhai, H.-O. Pörtner, D. Roberts, J. Skea, P.R. Shukla, A. Pirani, W. Moufouma-Okia, C. Péan, R. Pidcock, S. Connors, J.B.R. Matthews, Y. Chen, X. Zhou, M.I. Gomis, E. Lonnoy, T. Maycock, M. Tignor, and T. Waterfield (eds.)], 2018.

JANSSENS-MANHOUT, G.; PAROULO, P.; MARTELLI, S. Analysis of Greenhouse Gas Emission Trends and Drivers. A Survey of Techniques for Emission Decomposition and Econometric Trend Analysis. European Union, JRC Technical Reports, Report 1: Literature survey and project outlook, Bruxelas, 2013.

KAYA, Y., Impact of Carbon Dioxide Emission Control on GNP Growth: Interpretation of Proposed Scenarios. Paper presented to the IPCC Energy and Industry Subgroup, Response Strategies Working Group, Paris, (mimeo), 1990.

PLANO ABC. Plano Setorial de Mitigação e de Adaptação às Mudanças Climáticas para a Consolidação de uma Economia de Baixa Emissão de Carbono na Agricultura, Ministério da Agricultura, Pecuária e Abastecimento, 2012. Disponível em:

https://www.gov.br/agricultura/pt-br/assuntos/sustentabilidade/plano-abc/plano-abcagricultura-de-baixa-emissao-de-carbono, acesso em: 12/09/2020.

PLANOS DE PREVENÇÃO E CONTROLE DO DESMATAMENTO EM ÂMBITO FEDERAL. Ministério do Meio Ambiente, s.d. Disponível em: http://combateaodesmatamento.mma.gov.br/, acesso em: 12/09/2020.

RESENHA ENERGÉTICA BRASILEIRA, Exercício 2018, Ministério das Minas e Energia, Secretaria de Planejamento e Desenvolvimento Energético, edição de maio de 2019. Disponível em: http://www.mme.gov.br/web/guest/secretarias/planejamento-e-desenvolvimentoenergetico/publicacoes/resenha-energetica-brasileira, acesso em: 13/09/2020.

RITTL, C.; ANGELO, C. Is Brazil in the way to meet its climate targets? Observatório do Clima, 2019. Disponível em: http://www.observatoriodoclima.eco.br/explainer-brazil-way-meetclimate-targets/, acesso em: 12/09/2020.

SANDERS, B. The New Green Deal, s.d. Disponível em: https://berniesanders.com/issues/greennew-deal/, acesso em: 12/09/2020.

SEEG, Sistema de Estimativas de Emissões e Remoções de Gases de Efeito Estufa, 2019. Disponível em: http://seeg.eco.br/download, acesso em: 12/09/2020. 
WORLD BANK DATA, 2020. Base de dados disponível em:

https://data.worldbank.org/topic/climate-change?view=chart, acesso em: 02/09/2020. 